\title{
Characterization of accessibility for affine connection control systems at some points with nonzero velocity
}

\author{
María Barbero Liñán
}

\begin{abstract}
Affine connection control systems are mechanical control systems that model a wide range of real systems such as robotic legs, hovercrafts, planar rigid bodies, rolling pennies, snakeboards and so on. In 1997 the accessibility and a particular notion of controllability was intrinsically described by A. D. Lewis and R. Murray at points of zero velocity. Here, we present a novel generalization of the description of accessibility algebra for those systems at some points with nonzero velocity as long as the affine connection restricts to the distribution given by the symmetric closure. The results are used to describe the accessibility algebra of different mechanical control systems.
\end{abstract}

\section{INTRODUCTION}

A wide range of mechanical control systems can be described in terms of an affine connection on the configuration manifold $Q$ [1].

An analytic affine connection control system (ACCS) $\Sigma$ is a 4-tuple $(Q, \nabla, \mathscr{Y}, U)$ where

- $Q$ is a configuration manifold,

- $\nabla$ is an affine connection on $Q$,

- $\mathscr{Y}=\left\{Y_{1}, \ldots, Y_{r}\right\}$ is a set of vector fields on $Q$ socalled control or input vector fields,

- $U$ is a subset of $\mathbb{R}^{r}$.

The typical assumption on $U$ is to be an almost proper control set, that is, zero is in the convex hull of $U(0 \in$ $\operatorname{conv}(U))$ and the affine hull of $U$ is the entire Euclidean space $\mathbb{R}^{r}\left(\operatorname{aff}(U)=\mathbb{R}^{r}\right)$.

The dynamics are described by a second-order differential equation on $Q$

$$
\nabla_{\gamma^{\prime}(t)} \gamma^{\prime}(t)=u^{a} Y_{a}(\gamma(t))
$$

where $\gamma: I \subset \mathbb{R} \rightarrow Q, u: I \rightarrow U \subset \mathbb{R}^{r}$.

It is well-known the control system (I.1) is equivalent to a first-order control-affine system on $T Q$

$$
\Upsilon^{\prime}(t)=Z(\Upsilon(t))+u^{a} Y_{a}^{V}(\Upsilon(t)),
$$

where $\Upsilon: I \rightarrow T Q$ projects onto $Q$ to $\gamma$ through $\tau_{Q}: T Q \rightarrow$ $Q, Z$ is the geodesic spray and $Y_{a}^{V}$ is the vertical lift of the vector field $Y_{a}$ on $Q$ to $T Q$.

This work has been partially supported by MICINN (Spain) Grants MTM200800689 and MTM200908166; 2009SGR1338 of the Catalan government, IRSES project GEOMECH (246981) within the 7th European Community Framework Program, by Beatriu de Pinós fellowship from Comissionat per a Universitats i Recerca del Departament d'Innovació, Universitats i Empresa of Generalitat de Catalunya and by Juan de la Cierva fellowship from MICINN.

This work was mainly developed while a postdoctoral fellow at Queen's University, Kingston, ON, Canada. M. Barbero Liñán is with the Institute for the Mathematical Sciences (CSIC-UAM-UC3M-UCM), Madrid, Spain mbarberodicmat.es
One of the key objects to describe the accessibility and controllability of control-affine systems such as (I.2) is the involutive closure $\operatorname{Lie}^{\infty}\left(Z, \mathscr{Y}^{V}\right)$ of the vector fields in (I.2) and the "symmetric" closure $\operatorname{Sym}^{(\infty)}(\mathscr{Y})$ of $\mathscr{Y}$ that will be defined in Section [I-B In [5] the accessibility distribution was characterized at points of zero velocity. In this paper we are going to extend this characterization to points with velocity in $\operatorname{Sym}^{(\infty)}(\mathscr{Y})$ as long as $\nabla$ is restricted to $\operatorname{Sym}^{(\infty)}(\mathscr{Y})$, cf. Proposition 4.4 This will allow us to extend the characterization of accessibility for mechanical control systems in [5], cf. Theorem 4.5.

The paper is organized as follows. Section $\prod$ reviews the main properties and related results about accessibility of the mechanical control systems under study. It also introduces the notion of a connection which restricts to a distribution that will be an essential assumption for the results below. Sections IIII and IV contain all the novel results of this paper. Theorem 3.1 characterizes a family of generators of $\operatorname{Lie}^{(\infty)}\left(Z, \mathscr{Y}^{V}\right)$ at any $v_{q} \in T Q$. This family is not a minimal set of generators, but it provides a suitable set of generators for obtaining all the remaining results in this paper that culminate in Propositions 4.3, 4.4 and Theorem 4.5. Section $\mathrm{V}$ applies the above results to two mechanical control systems: the planar rigid body with variable-direction thruster and the rolling penny.

We shall assume all manifolds are paracompact, Hausdorff, and of class $\mathcal{C}^{\omega}$ (analytic). All maps and geometric objects will be assumed to be of class $\mathcal{C}^{\omega}$. The set of analytic functions on a manifold $Q$ is denoted by $\mathcal{C}^{\omega}(Q)$. For a manifold $Q$, its tangent bundle will be denoted by $\tau_{Q}: T Q \rightarrow Q$. If $\pi: E \rightarrow M$ is a vector bundle over $M$, we denote by $\Gamma^{\omega}(E)$ the set of analytic sections of $E$. By $0_{x} \in E_{x}$ we denote the zero vector in the fiber at $x$. The analyticity assumption is necessary to have sufficient and necessary characterizations of the accessibility algebra, cf. Theorems 2.2, 2.3, 4.5 The control set is always assumed to be almost proper.

\section{NOTATION AND PREVIOUS CONCEPTS}

This section contains the properties of control systems and some notions of differential geometry that would be necessary at some point in the development of this paper. This is not a detailed description. We refer to [1], [3], [4], [5], [8], [9] for more details.

\section{A. Properties of a control system}

Typical studied properties of a control system are accessibility and controllability. The former has been widely 
understood [8], [9], whereas the latter is still under study [1]. To define them formally we first need to introduce the notion of a reachable set $\mathcal{R}\left(v_{q}, T\right)$ from $v_{q} \in T Q$ at time $T \in \mathbb{R}^{+}$

$\mathcal{R}\left(v_{q}, T\right)=\left\{\begin{array}{l|l}w \in T Q & \begin{array}{l}\exists \text { a trajectory }(\Upsilon, u) \text { of (I.2) } \\ \text { such that } \Upsilon(0)=v_{q}, \\ \Upsilon(T)=w\end{array}\end{array}\right\}$

Then the reachable set from $v_{q}$ up to time $T$ is defined as follows

$$
\mathcal{R}\left(v_{q}, \leq T\right)=\bigcup_{t \in[0, T]} \mathcal{R}\left(v_{q}, t\right)
$$

These reachable sets collect the information about states and velocities that can be reached by trajectories of the control system (I.2). When we study a mechanical system, we might just be interested in the reachable states. That is why we define the reachable set $\mathcal{R}_{Q}(q, T)$ in $Q$ from $q$ at time $T$,

$$
\mathcal{R}_{Q}(q, T)=\left\{\begin{array}{l|l}
\bar{q} \in Q & \begin{array}{l}
\exists \text { a trajectory }(\Upsilon, u) \text { of } \\
\text { such that } \tau_{Q}(\Upsilon(0))=q, \\
\tau_{Q}(\Upsilon(T))=\bar{q}
\end{array}
\end{array}\right\} .
$$

As before, the reachable set $\mathcal{R}_{Q}(q, \leq T)$ in $Q$ from $v_{q}$ up to time $T$ is defined as follows

$$
\mathcal{R}_{Q}(q, \leq T)=\bigcup_{t \in[0, T]} \mathcal{R}_{Q}(q, t)
$$

Definition 2.1: Let $\Sigma$ be an analytic affine connection control system and let $v_{q} \in T Q$.

1) $\Sigma$ is accessible from $v_{q}$ if there exists $T>0$ such that

$$
\operatorname{int}\left(\mathcal{R}\left(v_{q}, \leq t\right)\right) \neq \emptyset \quad \text { for } t \in(0, T] .
$$

2) $\Sigma$ is configuration accessible from $q \in Q$ if there exists $T>0$ such that

$$
\operatorname{int}\left(\mathcal{R}_{Q}(q, \leq t)\right) \neq \emptyset \text { for each } t \in(0, T] .
$$

3) $\Sigma$ is small-time locally controllable (STLC) from $v_{q}$ if there exists $T>0$ such that

$$
v_{q} \in \operatorname{int}\left(\mathcal{R}\left(v_{q}, \leq t\right)\right) \text { for each } t \in(0, T] .
$$

4) $\Sigma$ is small-time locally configuration controllable (STLCC) from $v_{q}$ if there exists $T>0$ such that

$$
v_{q} \in \operatorname{int}\left(\mathcal{R}_{Q}\left(v_{q}, \leq t\right)\right) \text { for each } t \in(0, T]
$$

In this paper we only focus on extending the characterization of the accessibility algebra given in [5], but we include the definition of controllability for completeness of the paper.

\section{B. Characterization of accessibility algebra at zero velocity}

For any control-affine system on $Q$

$$
\dot{q}=f_{0}(q)+u^{a} f_{a}(q),
$$

the accessibility distribution at $q$ is the involutive closure at $q$ of the distribution generated by the drift vector field $f_{0}$ and the control vector fields $\left\{f_{a}\right\}_{a=1, \ldots, r}$.
Theorem 2.2 ([8], [9]): The control-affine system in (II.3 is accessible from $q$ if and only if $\operatorname{Lie}^{(\infty)}\left(f_{0}, f_{1}, \ldots, f_{r}\right)_{q} \simeq T_{q} Q$.

This same result can also be applied to the control system (I.2). Thus, $\Sigma$ is accessible from $v_{q} \in T Q$ if and only if $\mathrm{Lie}^{(\infty)}\left(Z, \mathscr{Y}^{V}\right)_{v_{q}} \simeq T_{v_{q}} T Q$. Observe that to define the accessibility algebra the control set does not play any role.

Let us review now the intrinsic description of the accessibility algebra at zero velocity given in [5]. At $0_{q}$ there exists the following natural isomorphism

$$
T_{0_{q}} T Q \simeq T_{q} Q \oplus T_{q} Q,
$$

\section{cf. [1, Lemma 6.33].}

Given an affine connection $\nabla$, there exists a complementary subbundle $H T Q$ of the vertical subbundle $V T Q=$ $\operatorname{ker}\left(T \tau_{Q}\right)$ such that $T T Q=H T Q \oplus V T Q$. This complementary subbundle is called horizontal subbundle. We refer to [3, page 87] for a characterization of this subbundle. Then the splitting in (II.4) corresponds with the splitting into horizontal and vertical subspace.

Remember that for an affine connection the symmetric product of two vector fields is defined as follows

$$
\langle X: Y\rangle=\nabla_{X} Y+\nabla_{Y} X
$$

Analogously to the involutive closure of vector fields, we define the symmetric closure $\operatorname{Sym}^{(\infty)}(\mathscr{Y})$ of a set $\mathscr{Y}$ of control vector fields as the distribution being the smallest $\mathbb{R}$ subspace of vector fields containing $\mathscr{Y}$ and closed under the symmetric product.

Theorem 2.3: [5, Lemma 5.7] Let $\Sigma$ be an ACCS. If $\mathcal{C}_{\Sigma}=$ $\left\{Z, \mathscr{Y}^{V}\right\}$, then, for $q \in Q$,

$$
\operatorname{Lie}^{(\infty)}\left(\mathcal{C}_{\Sigma}\right)_{0_{q}}=\operatorname{Lie}^{(\infty)}\left(\operatorname{Sym}^{(\infty)}(\mathscr{Y})\right)_{q} \oplus \operatorname{Sym}^{(\infty)}(\mathscr{Y})_{q} .
$$

Moreover:

1) $\Sigma$ is accessible from $0_{q}$ if and only if $\operatorname{Sym}^{(\infty)}(\mathscr{Y})_{q}=$ $T_{q} Q$.

2) $\Sigma$ is configuration accessible from $0_{q}$ if and only if $\operatorname{Lie}^{(\infty)}\left(\operatorname{Sym}^{(\infty)}(\mathscr{Y})\right)_{q}=T_{q} Q$.

\section{On connections restricted to distributions}

As mentioned above, the generalization of Theorem 2.3 in this paper is only possible under suitable assumptions, cf. Theorem 4.5 An affine connection $\nabla$ restricts to a distribution $\mathcal{D}$ if $\nabla_{X} Y$ is a section of $\mathcal{D}$ for every section $Y$ of $\mathcal{D}$ and $X \in \Gamma^{\omega}(T Q)$ [4].

Associated with an affine connection, we define the curvature of $\nabla$ as a $(1,3)$-tensor field on $Q$ such that

$$
R(X, Y) W=\nabla_{X} \nabla_{Y} W-\nabla_{Y} \nabla_{X} W-\nabla_{[X, Y]} W
$$

for $X, Y, W \in \Gamma^{\omega}(T Q)$.

Proposition 2.4: [4, Proposition 4.3] Let $q \in Q$ and let $u, v \in T_{q} Q$. If $\nabla$ restricts to $\mathcal{D}$, then the endomorphism $R(u, v)$ of $T_{q} Q$ leaves the subspace $\mathcal{D}_{q}$ invariant.

Another object of interest is the following one.

Definition 2.5: A distribution $\mathcal{D}$ on $Q$ is geodesically invariant under an affine connection $\nabla$ on $Q$ if, for every 
geodesic $\gamma: I \rightarrow Q$ for which $\gamma^{\prime}\left(t_{0}\right) \in \mathcal{D}_{\gamma\left(t_{0}\right)}$ for some $t_{0} \in I$, it holds that $\gamma^{\prime}(t) \in \mathcal{D}_{\gamma(t)}$ for every $t \in I$.

From the definition we have that a distribution $\mathcal{D}$ on $Q$ is geodesically invariant under an affine connection $\nabla$ on $Q$ if, as a submanifold of $T Q, \mathcal{D}$ is invariant under the geodesic spray associated with $\nabla$. One can then show that a distribution is geodesically invariant if and only if the symmetric product of any $\mathcal{D}$-valued vector fields is again a $\mathcal{D}$-valued vector field [4, Theorem 5.4].

Observe that if $\nabla$ restricts to a distribution $\mathcal{D}$, then $\mathcal{D}$ is geodesically invariant under $\nabla$.

\section{Primitive BRACKets}

A systematic way to construct the accessibility algebra is to compute iteratively the Lie brackets in $\operatorname{Lie}^{\infty}\left(Z, \mathscr{Y}^{V}\right)$. One of the key points to obtain the characterization of the accessibility algebra reviewed in Theorem 2.3 was to identify a family of primitive brackets that were enough to span the accessibility distribution at $0_{q}$. The novel result of this section is to identify the primitive brackets for the accessibility distribution at non-zero velocity. At zero velocity we recover the same primitive brackets as in [5].

Theorem 3.1: If $v_{q} \in T Q$, then any tangent vector in $\operatorname{Lie}^{\infty}\left(Z, \mathscr{Y}^{V}\right)_{v_{q}}$ is spanned by a linear combination of elements in:

1) $\left\{Z_{v_{q}}\right\}$;

2) $\mathcal{A}_{v_{q}}=\left(\operatorname{Sym}^{(\infty)}(\mathscr{Y})\right)_{v_{q}}^{V}$, i. e., the vertical lift of the smallest distribution containing $\mathscr{Y}$ and closed under the symmetric product;

3) $\mathcal{B}_{v_{q}}=\left\{\operatorname{ad}_{Z}^{l}\left(\operatorname{Sym}^{(\infty)}(\mathscr{Y})\right)_{v_{q}}^{V} \mid l \in \mathbb{N} \cup\{0\}\right\}$, i.e., the smallest distribution containing $\mathcal{A}$ and invariant under the geodesic spray;

4) $\mathcal{C}_{v_{q}}=\operatorname{Lie}^{(\infty)}\left(\left\{\operatorname{ad}_{Z}^{l}\left(\operatorname{Sym}^{(\infty)}(\mathscr{Y})\right)^{V} \mid l \in \mathbb{N}\right\}\right)_{v_{q}}$, i.e., the smallest involutive distribution containing $\mathcal{B}-\mathcal{A}$.

Proof: As stated in [5, Proposition 3.1] any vector field in $\operatorname{Lie}^{(\infty)}\left(Z, \mathscr{Y}^{V}\right)$ can be written as a linear combination of brackets of the form

$$
\left[W_{k},\left[W_{k-1},\left[\ldots,\left[W_{2}, W_{1}\right] \ldots\right]\right]\right]
$$

where $W_{i} \in\left\{Z, \mathscr{Y}^{V}\right\}, i=1, \ldots, k$. Thus we only need to prove this proposition for Lie brackets of this form.

Let us prove the result by induction on the length $k$ of the Lie brackets that generate the entire $\operatorname{Lie}^{(\infty)}\left(Z, \mathscr{Y}^{V}\right)$ at $v_{q}$.

For $k=1$, the only possible vector fields are $Z$ and $Y^{V}$. These vector fields live in the distributions of type 1 and 2 respectively.

We prove now the result for some Lie brackets of small length so that it is clear how to prove the induction step.

For $k=2$, the only nonzero vector field is $\left[Z, Y^{V}\right]$ that lives in the distribution of type 3.

For $k=3$, there are $\left[Z,\left[Z, Y^{V}\right]\right]$ and $\left[Y_{2}^{V},\left[Z, Y_{1}^{V}\right]\right]=$ $\left\langle Y_{2}: Y_{1}\right\rangle^{V}$ that live in the distribution of type 3 and 2 respectively.

For $k=4$, the vector fields are the following ones:

- $\left[Z,\left[Z,\left[Z, Y^{V}\right]\right]\right]=\operatorname{ad}_{Z}^{3}\left(Y^{V}\right)$ that lives in the distribution of type 3
- $\left[Y_{2}^{V},\left[Z,\left[Z, Y_{1}^{V}\right]\right]\right]$ is not that straightforward and we must use the Jacobi identity.

$$
\begin{aligned}
& {\left[Y_{2}^{V},\left[Z,\left[Z, Y_{1}^{V}\right]\right]\right]=-\left[\left[Z, Y_{1}^{V}\right],\left[Y_{2}^{V}, Z\right]\right.} \\
& \quad-\left[Z,\left[\left[Z, Y_{1}^{V}\right], Y_{2}^{V}\right]\right]=\left[\left[Z, Y_{1}^{V}\right],\left[Z, Y_{2}^{V}\right]\right] \\
& \quad+\left[Z,\left\langle Y_{2}: Y_{1}\right\rangle^{V}\right] .
\end{aligned}
$$

Thus the first summand is one of type 4 and the second one is of type 3 .

- $\left[Z,\left[Y_{2}^{V},\left[Z, Y_{1}^{V}\right]\right]\right]=\left[Z,\left\langle Y_{2}: Y_{1}\right\rangle^{V}\right]$ is of type 3.

- $\left[Y_{3}^{V},\left[Y_{2}^{V},\left[Z, Y_{1}^{V}\right]\right]\right]=\left[Y_{3}^{V},\left\langle Y_{2}: Y_{1}\right\rangle^{V}\right]=0$.

Now we have already given an idea how to rewrite the Lie brackets conveniently in order to express them as a linear combination of vector fields in any of the four types of vector fields described in the statement of Theorem 3.1. Let us prove the induction step.

Assume that any Lie bracket up to length $k$ can be written as a linear combination of vector fields among the four types mentioned in Theorem 3.1 then let us prove it is true for Lie brackets of length $k+1$.

By assumption any Lie bracket of length $k>1$ is either of type 2 or 3 or 4 . Then we only need to bracket these types of vector fields with $Z$ and the family of vertical vector fields $\mathscr{Y}^{V}$.

- $[Z, \mathcal{A}]$ is of type 3

- $[Z, \mathcal{B}]$ is still of type 3 .

- $[Z, \mathcal{C}]$ needs some more work.

A Lie bracket in $\mathcal{C}$ can be written as

$\left[\operatorname{ad}_{Z}^{k_{s}} W_{s}^{V},\left[\operatorname{ad}_{Z}^{k_{s-1}} W_{s-1}^{V},\left[\ldots,\left[\operatorname{ad}_{Z}^{k_{2}} W_{2}^{V}, \operatorname{ad}_{Z}^{k_{1}} W_{1}^{V}\right] \ldots\right]\right]\right]$, where $W_{i} \in \operatorname{Sym}^{(\infty)}(\mathscr{Y}), k_{i} \in \mathbb{N}, i=1, \ldots, s$.

We prove that $[Z, \mathcal{C}]$ can always be written as a linear combination of vector fields of type 3 and 4 by induction on the number of vertical vector fields involved in a vector field of type $\mathcal{C}$.

For $s=1$,

$$
\left[Z, \operatorname{ad}_{Z}^{k_{1}} W_{1}^{V}\right]=\operatorname{ad}_{Z}^{k_{1}+1} W_{1}^{V}
$$

is trivially of type 3 ,

Let us introduce the following notation to shorten the expressions:

$$
\begin{aligned}
\mathcal{V}_{i} & =\operatorname{ad}_{Z}^{k_{i}} W_{i}^{V}, \\
\mathcal{V}_{i j} & =\left[\operatorname{ad}_{Z}^{k_{j}} W_{j}^{V}, \operatorname{ad}_{Z}^{k_{i}} W_{i}^{V}\right]=\left[\mathcal{V}_{j}, \mathcal{V}_{i}\right], \\
& \ldots \\
\mathcal{V}_{i_{1} \ldots i_{s}} & =\left[\operatorname{ad}_{Z}^{k_{i s}} W_{i_{s}}^{V},\left[\ldots,\left[\operatorname{ad}_{Z}^{k_{i_{2}}} W_{i_{2}}^{V}, \operatorname{ad}_{Z}^{i_{1}} W_{i_{1}}^{V}\right] \ldots\right]\right] .
\end{aligned}
$$

For $s=2$,

$$
\begin{aligned}
{\left[Z, \mathcal{V}_{12}\right] } & =-\left[\mathcal{V}_{1},\left[Z, \mathcal{V}_{2}\right]\right]-\left[\mathcal{V}_{2},\left[\mathcal{V}_{1}, Z\right]\right] \\
& =\left[\operatorname{ad}_{Z}^{k_{2}+1} W_{2}^{V}, \mathcal{V}_{1}\right]+\left[\mathcal{V}_{2}, \operatorname{ad}_{Z}^{k_{1}+1} W_{1}^{V}\right] .
\end{aligned}
$$

Thus it is of type 4

The induction step is the following one for $s>2$ : If $\left[Z, \mathcal{V}_{i_{1} \ldots i_{s}}\right]$ can be written as a linear combination of vector fields of type 4 for any vector field $\mathcal{V}_{i_{1} \ldots i_{s}}$ in $\mathcal{C}$ 
with $s$ vertical vector fields involved, see (III.7), then $\left[Z, \mathcal{V}_{i_{1} \ldots i_{s+1}}\right]$ is a linear combination of vector fields of type 4 for any vector field $\mathcal{V}_{i_{1} \ldots i_{s+1}}$ in $\mathcal{C}$ with $s+1$ vertical vector fields involved.

$$
\begin{aligned}
{\left[Z, \mathcal{V}_{i_{1} \ldots i_{s+1}}\right]=\left[\operatorname{ad}_{Z}^{k_{s+1}+1} W_{s+1}^{V}, \mathcal{V}_{i_{1} \ldots i_{s}}\right] } \\
+\left[\operatorname{ad}_{Z}^{k_{s+1}} W_{s+1}^{V},\left[Z, \mathcal{V}_{i_{1} \ldots i_{s}}\right]\right]
\end{aligned}
$$

The first summand is of type 4 By induction hypothesis $\left[Z, \mathcal{V}_{i_{1} \ldots i_{s}}\right]$ can be expressed as a linear combination of vector fields of type 4. The induction concludes.

- $\left[Y^{V}, \mathcal{A}\right]=0$.

- $\left[Y^{V}, \mathcal{B}\right]$ needs some work. An element of $\mathcal{B}$ is given by $\operatorname{ad}_{Z}^{s}\left(W^{V}\right)$ where $W^{V} \in \mathcal{A}$, then

$$
\begin{aligned}
& {\left[Y^{V}, \operatorname{ad}_{Z}^{s}\left(W^{V}\right)\right]=} \\
& \quad-\left[\operatorname{ad}_{Z}^{s-1}\left(W^{V}\right),\left[Y^{V}, Z\right]\right]-\left[Z,\left[\operatorname{ad}_{Z}^{s-1}\left(W^{V}\right), Y^{V}\right]\right] \\
& \quad=\left[\operatorname{ad}_{Z}^{s-1}\left(W^{V}\right),\left[Z, Y^{V}\right]\right]+\left[Z,\left[Y^{V}, \operatorname{ad}_{Z}^{s-1}\left(W^{V}\right)\right]\right] .
\end{aligned}
$$

The first summand is of type 4. For the second one we need to do further study. By induction on $s$ $\left[Y^{V}, \operatorname{ad}_{Z}^{s-1}\left(W^{V}\right)\right]$ can be written as a linear combination of vector fields of type 2,3 and 4 As the bracketing of $Z$ with any of these three types of distributions is of type 3 and 4 according to the first cases considered, our reasoning concludes.

- $\left[Y^{V}, \mathcal{C}\right]$ needs some more work. As in the case $[Z, \mathcal{C}]$, we consider induction on the number $s$ of vertical lift of vector fields in $\mathcal{C}$ in order to prove that all the vector fields in $\left[Y^{V}, \mathcal{C}\right]$ can be expressed as a linear combination of vector fields of type 4

For $s=1$, we have $\left[Y^{V}, \operatorname{ad}_{Z}^{k_{1}} W_{1}^{V}\right]$.

We prove it by induction on $k_{1}$. For $k_{1}=1$,

$$
\left[Y^{V}, \operatorname{ad}_{Z}^{1} W_{1}^{V}\right]=\left\langle Y: W_{1}\right\rangle^{V} .
$$

By induction we prove that for $k_{1}>1$

$$
\begin{aligned}
& {\left[Y^{V}, \operatorname{ad}_{Z}^{k_{1}} W_{1}^{V}\right]=\left(k_{1}-1\right)\left[\operatorname{ad}_{Z}^{k_{1}-1}\left(W_{1}^{V}\right), \operatorname{ad}_{Z}^{1}\left(Y^{V}\right)\right]} \\
& \quad+\sum_{j=1}^{k_{1}-2} A_{j}^{\left(k_{1}\right)}\left[\operatorname{ad}_{Z}^{j}\left(W_{1}^{V}\right), \operatorname{ad}_{Z}^{k_{1}-j}\left(Y^{V}\right)\right] \\
& \quad+\operatorname{ad}_{Z}^{k_{1}-1}\left(\left\langle Y: W_{1}\right\rangle^{V}\right) \\
& \quad=\sum_{j=1}^{k_{1}-1} A_{j}^{\left(k_{1}\right)}\left[\operatorname{ad}_{Z}^{j}\left(W_{1}^{V}\right), \operatorname{ad}_{Z}^{k_{1}-j}\left(Y^{V}\right)\right] \\
& \quad+\operatorname{ad}_{Z}^{k_{1}-1}\left(\left\langle Y: W_{1}\right\rangle^{V}\right),
\end{aligned}
$$

where $A_{j}^{\left(k_{1}\right)} \in \mathbb{N}$ satisfies

$$
\begin{aligned}
A_{0}^{\left(k_{1}\right)} & =1, \\
A_{1}^{\left(k_{1}\right)} & =A_{1}^{\left(k_{1}-1\right)}, \\
A_{j}^{\left(k_{1}\right)} & =A_{j}^{\left(k_{1}-1\right)}+A_{j-1}^{\left(k_{1}-1\right)}, \quad \text { for } 2 \leq \mathrm{j} \leq \mathrm{k}_{1}-2, \\
A_{k_{1}-1}^{\left(k_{1}\right)} & =k_{1}-1 .
\end{aligned}
$$

For $k_{1}=2$,

$$
\begin{aligned}
{\left[Y^{V},\right.} & \left.\operatorname{ad}_{Z}^{2} W_{1}^{V}\right]= \\
& -\left[\left[Z, W_{1}^{V}\right],\left[Y^{V}, Z\right]\right]-\left[Z,\left[\left[Z, W_{1}^{V}\right], Y^{V}\right]\right] \\
& =\left[\left[Z, W_{1}^{V}\right],\left[Z, Y^{V}\right]\right] \\
& +\left[Z,\left\langle Y: W_{1}\right\rangle^{V}\right] .
\end{aligned}
$$

Assume it is true for $k_{1}$, let us prove it for $k_{1}+1$.

$$
\begin{aligned}
& {\left[Y^{V}, \operatorname{ad}_{Z}^{k_{1}+1} W_{1}^{V}\right]=-\left[\operatorname{ad}_{Z}^{k_{1}} W_{1}^{V},\left[Y^{V}, Z\right]\right]} \\
& -\left[Z,\left[\operatorname{ad}_{Z}^{k_{1}} W_{1}^{V}, Y^{V}\right]\right]=\left[\operatorname{ad}_{Z}^{k_{1}} W_{1}^{V},\left[Z, Y^{V}\right]\right] \\
& +\left[Z,\left[\left(k_{1}-1\right)\left[\operatorname{ad}_{Z}^{k_{1}-1}\left(W_{1}^{V}\right), \operatorname{ad}_{Z}^{1}\left(Y^{V}\right)\right]\right]\right] \\
& +\sum_{j=1}^{k_{1}-2} A_{j}^{\left(k_{1}\right)}\left[Z,\left[\operatorname{ad}_{Z}^{j}\left(W_{1}^{V}\right), \operatorname{ad}_{Z}^{k_{1}-j}\left(Y^{V}\right)\right]\right] \\
& +\operatorname{ad}_{Z}^{k_{1}}\left(\left\langle Y: W_{1}\right\rangle^{V}\right)=\left[\operatorname{ad}_{Z}^{k_{1}} W_{1}^{V},\left[Z, Y^{V}\right]\right] \\
& -\left(k_{1}-1\right)\left[\operatorname{ad}_{Z}^{1}\left(Y^{V}\right),\left[Z, \operatorname{ad}_{Z}^{k_{1}-1}\left(W_{1}^{V}\right)\right]\right] \\
& -\left(k_{1}-1\right)\left[\operatorname{ad}_{Z}^{k_{1}-1}\left(W_{1}^{V}\right),\left[\operatorname{ad}_{Z}^{1}\left(Y^{V}\right), Z\right]\right] \\
& -\sum_{j=1}^{k_{1}-2} A_{j}^{\left(k_{1}\right)}\left[\operatorname{ad}_{Z}^{k_{1}-j}\left(Y^{V}\right),\left[Z, \operatorname{ad}_{Z}^{j}\left(W_{1}^{V}\right)\right]\right] \\
& -\sum_{j=1}^{k_{1}-2} A_{j}^{\left(k_{1}\right)}\left[\operatorname{ad}_{Z}^{j}\left(W_{1}^{V}\right),\left[\operatorname{ad}_{Z}^{k_{1}-j}\left(Y^{V}\right), Z\right]\right] \\
& +\operatorname{ad}_{Z}^{k_{1}}\left(\left\langle Y: W_{1}\right\rangle^{V}\right)=k_{1}\left[\operatorname{ad}_{Z}^{k_{1}} W_{1}^{V},\left[Z, Y^{V}\right]\right] \\
& +A_{j}^{\left(k_{1}+1\right)} \sum_{j=1}^{k_{1}-1}\left[\operatorname{ad}_{Z}^{j}\left(W_{1}^{V}\right), \operatorname{ad}_{Z}^{k_{1}-j+1}\left(Y^{V}\right)\right] \\
& +\operatorname{ad}_{Z}^{k_{1}}\left(\left\langle Y: W_{1}\right\rangle^{V}\right)
\end{aligned}
$$

Thus it is a linear combination of vector of type 3 and 4

For $s=2$, according to the notation introduced in (III.5) and (III.6):

$$
\begin{aligned}
{\left[Y^{V}, \mathcal{V}_{12}\right] } & =-\left[\mathcal{V}_{1},\left[Y^{V}, \mathcal{V}_{2}\right]\right]-\left[\mathcal{V}_{2},\left[\mathcal{V}_{1}, Y^{V}\right]\right] \\
& =-\left[\mathcal{V}_{1},\left[Y^{V}, \mathcal{V}_{2}\right]\right]+\left[\mathcal{V}_{2},\left[Y^{V}, \mathcal{V}_{1}\right]\right]
\end{aligned}
$$

By the above induction result we know that both summands in the last equality are of type 4. Then we are done.

Consider now the induction step. Assume it is true for $s \geq 3$ that the vector fields in $\left[Y^{V}, \mathcal{C}\right]$ can be rewritten as linear combination of vector fields of type 4 let us prove it for $s+1$.

$$
\begin{aligned}
& {\left[Y^{V}, \mathcal{V}_{i_{1} \ldots i_{s+1}}\right]=} \\
& \quad-\left[\mathcal{V}_{i_{1} \ldots i_{s}},\left[Y^{V}, \mathcal{V}_{i_{s+1}}\right]\right]-\left[\mathcal{V}_{i_{1} \ldots i_{s+1}}, Y^{V}\right] \\
& \quad=-\left[\mathcal{V}_{i_{1} \ldots i_{s}},\left[Y^{V}, \mathcal{V}_{i_{s+1}}\right]\right]+\left[\mathcal{V}_{i_{s+1}},\left[Y^{V}, \mathcal{V}_{i_{1} \ldots i_{s}}\right]\right]
\end{aligned}
$$

Both summands are of type 4 because $\left[Y^{V}, \mathcal{V}_{s}\right]$, $\left[Y^{V}, \mathcal{V}_{i_{1} \ldots i_{s}}\right]$ are of type 4 by induction. Now the proof by induction is concluded.

Immediate from this result, we have the following description of the accessibility algebra at some points with non-zero velocity. 
Corollary 3.2: If $v_{q} \in \operatorname{Sym}^{(\infty)}(\mathscr{Y})$, then

$$
\operatorname{Lie}^{(\infty)}\left(Z, \mathscr{Y}^{V}\right)_{v_{q}} \subseteq T_{v_{q}} \operatorname{Sym}^{(\infty)}(\mathscr{Y}) .
$$

Proof: Note that

$$
\operatorname{Lie}^{(\infty)}\left(Z, \mathscr{Y}^{V}\right)_{v_{q}}=\operatorname{Lie}^{(\infty)}\left(Z,\left(\operatorname{Sym}^{(\infty)}(\mathscr{Y})\right)^{V}\right)_{v_{q}},
$$

because

$$
\mathscr{Y}_{q} \subseteq \operatorname{Sym}^{(\infty)}(\mathscr{Y})_{q}
$$

and

$$
\operatorname{Sym}^{(\infty)}(\mathscr{Y})_{v_{q}}^{V} \subseteq \operatorname{Lie}^{(\infty)}\left(Z, \mathscr{Y}^{V}\right)_{v_{q}}
$$

since $\left\langle Y_{a}: Y_{b}\right\rangle^{V}=\left[Y_{a}^{V},\left[Z, Y_{b}^{V}\right]\right]$.

As $\operatorname{Sym}^{(\infty)}(\mathscr{Y})$ is geodesically invariant by definition, the geodesic spray is tangent to $\operatorname{Sym}^{(\infty)}(\mathscr{Y})$, cf. Section II-C Thus, on $\operatorname{Sym}^{(\infty)}(\mathscr{Y})$ all the vector fields of type 3 in Theorem 3.1 are also tangent to $\operatorname{Sym}^{(\infty)}(\mathscr{Y})$. Then all the vector fields of type 4 in Theorem 3.1 are also tangent to $\operatorname{Sym}^{(\infty)}(\mathscr{Y})$ because of the definition of Lie bracket.

\section{DESCRIPTION OF THE ACCESSIBILITY ALGEBRA}

We are going to introduce here how to compute inductively and explicitly the vector fields in $\operatorname{Lie}^{\infty}\left(Z, \mathscr{Y}^{V}\right)$ since they would be necessary later on. At zero velocity there are many objects that vanish as described in [5], but at nonzero velocity the computations become more involved and it is useful to consider objects defined along the projection map $\tau_{Q}: T Q \rightarrow Q$ as described in [6], [7].

Given a connection $\nabla$ on $Q$, at any $v_{q} \in T Q$ there exists a pointwise splitting of $T_{v_{q}} T Q$ analogous to the one in (II.4).

$$
T_{v_{q}} T Q \simeq H_{v_{q}}(T Q) \oplus V_{v_{q}}(T Q) \simeq T_{q} Q \oplus T_{q} Q .
$$

For any vector field on $T Q$, the horizontal and the vertical projector at $v_{q} \in T Q$ are denoted by $\operatorname{hor}_{v_{q}}: T_{v_{q}} T Q \rightarrow$ $H_{v_{q}}(T Q) \simeq T_{q} Q, \operatorname{ver}_{v_{q}}: T_{v_{q}} T Q \rightarrow V_{v_{q}}(T Q) \simeq T_{q} Q$, respectively.

Remember that the vertical lift of a vector field $X$ on $Q$ is the vector field $X^{V}$ on $T Q$ defined by $X^{V}\left(v_{q}\right)=$ vlft $_{v_{q}}(X(q))$, where $\operatorname{vlft}_{v_{q}}: T_{q} Q \rightarrow T_{v_{q}} T Q$ is given by

$$
\operatorname{vlft}_{v_{q}}\left(X_{q}\right)=\left.\frac{\mathrm{d}}{\mathrm{d} t}\right|_{t=0}\left(v_{q}+t X_{q}\right)
$$

for any $v_{q} \in T Q$.

The horizontal lift of the tangent vector $v_{q} \in T_{q} Q$ at point $q \in Q$ is the tangent vector in $T_{v_{q}} T Q$ given by

$$
\operatorname{hlft}_{q}\left(v_{q}\right)=\left(T_{v_{q}} \tau_{Q} \mid H_{v_{q}} T Q\right)^{-1}\left(v_{q}\right) .
$$

Then, the horizontal lift of the vector field $X \in \Gamma^{\omega}(T Q)$ is the vector field $X^{H}$ in $\Gamma^{\omega}(T T Q)$ defined by $X^{H}\left(v_{q}\right)=$ $\operatorname{hlft}_{q}\left(X\left(\tau_{Q}\left(v_{q}\right)\right)\right)$.

Locally, if $X=X^{i} \partial / \partial q^{i}$, then

$$
\begin{aligned}
& X^{H}\left(v_{q}\right)=X^{i}(q)\left(\frac{\partial}{\partial q^{i}}-\Gamma_{i k}^{j}(q) v^{k} \frac{\partial}{\partial v^{j}}\right), \\
& X^{V}\left(v_{q}\right)=X^{i}(q) \frac{\partial}{\partial v^{i}} .
\end{aligned}
$$

It is possible to rewrite Lie brackets in $\operatorname{Lie}^{(\infty)}\left(Z, \mathscr{Y}^{V}\right)$ in terms of the splitting in (IV.8). The computation of the vector fields in $\mathrm{Lie}^{(\infty)}\left(Z, \mathscr{Y}^{V}\right)$ can be restricted to the brackets of the form

$$
\left[X_{k},\left[X_{k-1},\left[\ldots,\left[X_{2}, X_{1}\right] \ldots\right]\right]\right]
$$

cf. [5, Proposition 3.1]. For vector fields $X$ and $W$ on $Q$, it can be computed that

$$
\left[Z, X^{H}+W^{V}\right]=\left(\nabla_{v} X-W\right) \oplus\left(-R(X, v) v+\nabla_{v} W\right),
$$

$\left[Y^{V}, X^{H}+W^{V}\right]=0 \oplus\left(-\nabla_{X} Y\right)$,

where $R$ is the curvature tensor associated with $\nabla$ and $v$ denotes the vector field Id: $T Q \rightarrow T Q$ defined along the projection $\tau_{Q}: T Q \rightarrow Q$.

The horizontal and vertical lift are defined analogously for vector fields $X$ defined along the projection $\tau_{Q}: T Q \rightarrow Q$, that is, $\tau_{Q} \circ X=\tau_{Q}$. The vector fields with the smallest length in $\operatorname{Lie}^{(\infty)}\left(Z, \mathscr{Y}^{V}\right)$ can be rewritten as follows

$$
\begin{aligned}
{\left[Z, Y^{V}\right] } & =-Y \oplus \nabla_{v} Y, \\
{\left[Y_{1}^{V},\left[Z, Y_{2}^{V}\right]\right] } & =0 \oplus\left\langle Y_{1}: Y_{2}\right\rangle, \\
{\left[Z,\left[Z, Y^{V}\right]\right] } & =-2 \nabla_{v} Y \\
& \oplus\left(R(Y, v) v+\operatorname{ver}\left(\nabla_{v^{H}}^{H}\left(\nabla_{v} Y\right)^{V}\right)\right),
\end{aligned}
$$

where $\nabla^{H}$ denotes the horizontal lift of a connection defined on [10]. Note that for coordinates $\left(q^{i}, v^{i}\right)$ the only non-zero Christoffel symbols ${ }^{H} \Gamma_{j i}^{j}$ of $\nabla^{H}$ needed for our computations are:

$$
{ }^{H} \Gamma_{j \bar{i}}^{\bar{l}}={ }^{H} \Gamma_{\bar{j} i}^{\bar{l}}=\Gamma_{j i}^{l}
$$

where $i$ is the index corresponding to $q^{i}$ and $\bar{i}$ is the index corresponding to $v^{i}$. From here it easily follows $\nabla_{X^{H}}^{H} Y^{V}\left(v_{q}\right) \in V_{v_{q}} T Q$.

Note that in (IV.11) and IV.12) there are vector fields depending on the velocities. To compute the iterated Lie bracket from here we must use

$$
\begin{aligned}
{\left[Z, X^{H}+W^{V}\right]\left(v_{q}\right) } & =\left(\operatorname{ver}_{v_{q}}\left(\nabla_{v^{H}}^{H} X^{V}\right)-W\right) \\
& \oplus\left(-R(X, v) v+\operatorname{ver}_{v_{q}}\left(\nabla_{v^{H}}^{H} W^{V}\right)\right), \\
{\left[Y^{V}, X^{H}+W^{V}\right]\left(v_{q}\right) } & =\operatorname{ver}_{v_{q}}\left(\nabla_{Y^{V}}^{H} X^{V}\right) \\
\oplus & \left(\operatorname{ver}_{v_{q}}\left(\nabla_{Y^{V}}^{H} W^{V}+\nabla_{X^{H}}^{H} Y^{V}\right)\right) .
\end{aligned}
$$

instead of (IV.9) and (IV.10). Observe that in IV.14 and (IV.15) the vertical projector is always acting over vertical vector fields because of the definition of the horizontal lift $\nabla^{H}$ of $\nabla$.

\section{A. On the tangent space to a distribution}

It would be very useful to have a decomposition of the tangent space to a distribution in terms of the horizontal and vertical subspace. However this is only possible at points with zero velocity or if the connection restricts to the distribution, cf. Section II-C, as proved in the following proposition. 
Proposition 4.1: If $\nabla$ restricts to a distribution $\mathcal{D}$, then

$$
\begin{aligned}
\mathrm{T}_{v_{q}} \mathcal{D} \cap H_{v_{q}}(T Q) & \simeq T_{q} Q, \\
\mathrm{~T}_{v_{q}} \mathcal{D} \cap V_{v_{q}}(T Q) & \simeq \mathcal{D}_{q},
\end{aligned}
$$

for every $v_{q} \in \mathcal{D}$.

Proof: Let $k$ be the rank of the distribution $\mathcal{D}$, consider a $\mathcal{D}$-adapted basis $\left\{Y_{1}, \ldots, Y_{n}\right\}$ on $Q$ such that

$$
\operatorname{span}_{\mathbb{R}}\left\{Y_{1}(q), \ldots, Y_{k}(q)\right\}=\mathcal{D}_{q}, \quad \text { for every } q \in Q .
$$

The Christoffel symbols associated with the $\mathcal{D}$-adapted basis $\left\{Y_{1}, \ldots, Y_{n}\right\}$ are given by

$$
\nabla_{Y_{i}} Y_{j}=\Gamma_{i j}^{l} Y_{l}
$$

As $\nabla$ restricts to $\mathcal{D}$, we have

$$
\Gamma_{i a}^{\alpha}=0
$$

for $\alpha=k+1, \ldots, n ; a=1, \ldots, k ; i=1, \ldots, n$. Then the basis of the horizontal subspace of $T_{v_{q}} T Q$ on $\mathcal{D}$ is tangent to $\mathcal{D}$ since

$$
Y_{i}^{H}=Y_{i}-\Gamma_{i j}^{l} v^{j} Y_{l}^{V}=Y_{i}-\Gamma_{i b}^{c} v^{b} Y_{c}^{V},
$$

for $i=1, \ldots, n ; b, c=1, \ldots, k$.

The tangency of vector fields on $T Q$ can be intrinsically characterized as follows. We let $\pi_{\mathcal{D}}: T Q \rightarrow T Q / \mathcal{D}$ be the canonical projection such that the following diagram commutes

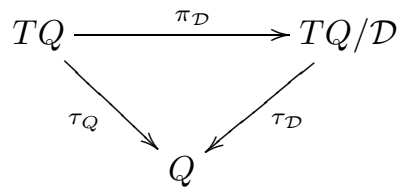

At $0_{q} \in T Q / \mathcal{D}$, there exists the following natural splitting

$$
T_{0_{q}} T Q / \mathcal{D} \simeq T_{q} Q \oplus\left(T_{q} Q / \mathcal{D}_{q}\right)
$$

cf. [1, Lemma 6.33]. Hence we define the projection $\pi_{2}: T_{0_{q}} T Q / \mathcal{D} \rightarrow T_{q} Q / \mathcal{D}_{q}$ onto the second component of the splitting in (IV.16). The vector field $Y_{i}^{H}$ on $T Q$ is tangent to $\mathcal{D}$ if and only if

$$
\pi_{2}\left(\left(T_{v_{q}} \pi_{\mathcal{D}} \circ Y_{i}^{H}\right)\left(v_{q}\right)\right)=0_{q}
$$

for every $v_{q} \in \mathcal{D}$. This equation is true because it is wellknown that $Y_{c}^{V}$ is tangent to $\mathcal{D}$ for $c=1, \ldots, k$. The result follows from here.

Now, under suitable assumptions, we are going to describe intrinsically the distribution of vector fields of type 3 in Theorem 3.1. First we need the following result.

Proposition 4.2: If $\nabla$ restricts to a distribution $\mathcal{D}$, then $\nabla^{H}$ restricts to the distribution $\mathcal{D}^{V}$.

Proof: It follows from IV.13 and from considering a $\mathcal{D}$-adapted basis on $Q$. To be more precise, for any section $C^{a} Y_{a}^{V}$ of $\mathcal{D}^{V}$

$$
\begin{aligned}
\nabla_{A^{i} Y_{i}+B^{i} Y_{i}^{V}}^{H}\left(C^{a} Y_{a}^{V}\right) & =\left(A^{i} Y_{i}+B^{i} Y_{i}^{V}\right)\left(C^{a}\right) Y_{a}^{V} \\
& +\Gamma_{i c}^{a} A^{i} C_{c} Y_{a}^{V} \in \Gamma^{\infty}\left(\mathcal{D}^{V}\right) .
\end{aligned}
$$

Proposition 4.3: If $\nabla$ restricts to a distribution $\mathcal{D}$, then the smallest distribution $\mathcal{D}^{Z}$ invariant under the geodesic spray that contains $\mathcal{D}$ satisfies

$$
\mathcal{D}^{Z}\left(v_{q}\right) \simeq \mathcal{D}_{q} \oplus \mathcal{D}_{q}
$$

for every $v_{q} \in \mathcal{D}$.

Proof: Let us prove it by induction on the number $s$ of iterated Lie brackets with the geodesic spray.

For $s=1$, see (IV.11). As $\nabla$ restricts to $\mathcal{D}$, the vertical component of (IV.11) sits in $\mathcal{D}$.

For $s=2$, see (IV.12). As $\nabla$ restricts to $\mathcal{D}$, the curvature tensor leaves $\mathcal{D}$ invariant, cf. Proposition 2.4. Using also Proposition 4.2 we have $\left[Z,\left[Z, Y^{V}\right]\right]\left(v_{q}\right) \in \mathcal{D}_{q} \oplus \mathcal{D}_{q}$ for every $v_{q} \in \mathcal{D}$.

Assume the result is true for $s$, let us prove it for $s+1$. Consider the following Lie bracket

$$
\begin{aligned}
{\left[Z, X^{H}+W^{V}\right]\left(v_{q}\right) } & =\left(\operatorname{ver}_{\mathrm{v}_{\mathrm{q}}}\left(\nabla_{\mathrm{v}^{\mathrm{H}}}^{\mathrm{H}} \mathrm{X}^{\mathrm{V}}\right)-\mathrm{W}\right) \\
& \oplus\left(-R(X, v) v+\operatorname{ver}_{\mathrm{v}_{\mathrm{q}}}\left(\nabla_{\mathrm{v}^{\mathrm{H}}}^{\mathrm{H}} \mathrm{W}^{\mathrm{V}}\right)\right),
\end{aligned}
$$

where $\left.X\right|_{\mathcal{D}},\left.W\right|_{\mathcal{D}}$ are $\mathcal{D}$-valued vector fields along $\tau_{Q}$ and ver $_{\mathrm{v}_{\mathrm{q}}}$ is the projection onto the vertical subspace according to the splitting in (IV.8). Thus the vertical lifts of $X$ and $W$ are sections of $\mathcal{D}^{V}$. By Proposition 4.2 we conclude that both $\nabla_{v^{H}}^{H} X^{V}$ and $\nabla_{v^{H}}^{H} W^{V}$ are vector fields tangent to $\mathcal{D}$. Moreover they are vertical vector fields, then their vertical projections are sections of $\mathcal{D}$. As the curvature tensor leaves $\mathcal{D}$ invariant, cf. Proposition 2.4 , the result follows.

Proposition 4.4: If $\nabla$ restricts to $\operatorname{Sym}^{(\infty)}(\mathscr{Y})$, then

$$
\begin{array}{r}
\operatorname{Sym}^{(\infty)}(\mathscr{Y})_{q} \simeq \operatorname{Lie}^{(\infty)}\left(Z, \mathscr{Y}^{V}\right)_{v_{q}} \cap V_{v_{q}}(T Q), \\
\operatorname{Lie}^{(\infty)}\left(\operatorname{Sym}^{(\infty)}(\mathscr{Y})\right)_{q} \subseteq \operatorname{Lie}^{(\infty)}\left(Z, \mathscr{Y}^{V}\right)_{v_{q}} \cap H_{v_{q}}(T Q),
\end{array}
$$

for every $v_{q} \in \operatorname{Sym}^{(\infty)}(\mathscr{Y})$.

Proof: Remember that

$$
\operatorname{Lie}^{(\infty)}\left(Z, \mathscr{Y}^{V}\right)_{v_{q}}=\operatorname{Lie}^{(\infty)}\left(Z, \operatorname{Sym}^{(\infty)}(\mathscr{Y})^{V}\right) v_{q} .
$$

The hypothesis, Corollary 3.2 and Proposition 4.1 leads to

$$
\begin{aligned}
\operatorname{Lie}^{(\infty)}\left(Z, \operatorname{Sym}^{(\infty)}(\mathscr{Y})^{V}\right)_{v_{q}} & \subseteq T_{v_{q}} \operatorname{Sym}^{(\infty)}(\mathscr{Y}) \\
& \simeq T_{q} Q \oplus \operatorname{Sym}^{(\infty)}(\mathscr{Y})_{q} .
\end{aligned}
$$

Since $\operatorname{Sym}^{(\infty)}(\mathscr{Y})_{v_{q}}^{V}$ sits in $\operatorname{Lie}^{(\infty)}\left(Z, \operatorname{Sym}^{(\infty)}(\mathscr{Y})^{V}\right)_{v_{q}}$ for every $v_{q} \in T Q$,

$$
\operatorname{Sym}^{(\infty)}(\mathscr{Y})_{q} \simeq \operatorname{Lie}^{(\infty)}\left(Z, \mathscr{Y}^{V}\right)_{v_{q}} \cap V_{v_{q}}(T Q) .
$$

To prove (IV.18) first note that by Theorem 3.1 and Proposition 4.3

$$
\operatorname{Sym}^{(\infty)}(\mathscr{Y})_{q} \subseteq \operatorname{Lie}^{(\infty)}\left(Z, \mathscr{Y}^{V}\right)_{v_{q}} \cap H_{v_{q}}(T Q) .
$$

Using (IV.11 and (IV.9), for every $Y_{1}, Y_{2} \in$ $\Gamma^{\omega}\left(\operatorname{Sym}^{(\infty)}(\mathscr{Y})\right)$ we have

$$
\left[\left[Z, Y_{1}^{V}\right],\left[Z, Y_{2}^{V}\right]\right]\left(v_{q}\right)=\left[Y_{1}, Y_{2}\right]_{q} \oplus W_{q},
$$

for every $v_{q} \in \operatorname{Sym}^{(\infty)}(\mathscr{Y})$ where $W \in \Gamma^{\omega}\left(\operatorname{Sym}^{(\infty)}(\mathscr{Y})\right)$ because of (IV.17). From here (IV.18) follows. 
Theorem 4.5: Let $\Sigma$ be an analytic ACCS. If $\nabla$ restricts to $\operatorname{Sym}^{(\infty)}(\mathscr{Y})$, then

1) $\Sigma$ is accessible from $v_{q} \in \operatorname{Sym}^{(\infty)}(\mathscr{Y})$ if and only if

$$
\begin{aligned}
\operatorname{Sym}^{(\infty)}(\mathscr{Y})_{q} & =T_{q} Q \\
\operatorname{Lie}^{(\infty)}\left(Z, \mathscr{Y}^{V}\right)_{v_{q}} & =T_{v_{q}} \operatorname{Sym}^{(\infty)}(\mathscr{Y}) .
\end{aligned}
$$

2) $\Sigma$ is configuration accessible from $v_{q} \in \operatorname{Sym}^{(\infty)}(\mathscr{Y})$ if and only if

$$
\operatorname{Lie}^{(\infty)}\left(Z, \mathscr{Y}^{V}\right)_{v_{q}}=T_{v_{q}} \operatorname{Sym}^{(\infty)}(\mathscr{Y})
$$

Proof: The proof follows from the proof of Theorem 2.2 and Theorem 2.3 using (IV.17) in Proposition 4.4

Moreover, if $\operatorname{Lie}_{v_{q}}^{(\infty)}\left(Z, \mathscr{Y}^{V}\right)=T_{v_{q}} \operatorname{Sym}^{(\infty)}(\mathscr{Y})$, then horizontal subspace of $\operatorname{Lie}_{v_{q}}^{(\infty)}\left(Z, \mathscr{Y}^{V}\right)$ at $v_{q} \in \operatorname{Sym}^{(\infty)}(\mathscr{Y})$ is the entire tangent space $T_{q} Q$.

\section{EXAMPLES}

Let us use Theorem 4.5 to describe the accessibility of some mechanical controls systems defined by an affine connection from points with nonzero velocity. These examples are more thoroughly described in [1, Section 7.4.2-3].

\section{A. Planar body with variable-direction thruster}

The configuration manifold for the system is $Q=\mathbb{S}^{1} \times$ $\mathbb{R}^{2}$. We denote the coordinates by $(\theta, x, y)$. The Riemannian metric for the system is

$$
\mathbb{G}=J \mathrm{~d} \theta \otimes \mathrm{d} \theta+m(\mathrm{~d} x \otimes \mathrm{d} x+\mathrm{d} y \otimes \mathrm{d} y),
$$

where $m$ is the mass of the body and $J$ is its moment of inertia about its center of mass. Then the Christoffel symbols are all zero.

This systems has two input vector fields given by

$$
\begin{aligned}
& Y_{1}=\frac{\cos \theta}{m} \frac{\partial}{\partial x}+\frac{\sin \theta}{m} \frac{\partial}{\partial y} \\
& Y_{2}=-\frac{h}{J} \frac{\partial}{\partial \theta}-\frac{\sin \theta}{m} \frac{\partial}{\partial x}+\frac{\cos \theta}{m} \frac{\partial}{\partial y} .
\end{aligned}
$$

This system is an affine connection control system and we can study its accessibility from nonzero velocities in $\operatorname{Sym}^{(\infty)} \mathscr{Y}$. As $\operatorname{Sym}^{(\infty)} \mathscr{Y}_{q}=T_{q} Q$, it is trivial that $\nabla$ restricts to $\operatorname{Sym}^{(\infty)} \mathscr{Y}_{q}$. Moreover, $\operatorname{Lie}^{(\infty)}\left(\operatorname{Sym}^{(\infty)} \mathscr{Y}\right)_{q} \simeq$ $T_{q} Q$. Having in mind Proposition 4.1, Proposition 4.4 and Theorem 4.5, we can conclude that the system is accessible and configuration accessible from $v_{q} \in \operatorname{Sym}^{(\infty)} \mathscr{Y}$.

\section{B. Rolling disk}

The configuration manifold is $Q=\mathbb{R}^{2} \times \mathbb{S}^{1} \times \mathbb{S}^{1}$. The coordinates are denoted by $(x, y, \theta, \phi)$. This system has constraints and to study the accessibility of the system the constrained connection in [1, Section 4.5.5] will have to be considered. The configuration manifold then is restricted to $\bar{Q}$ with dimension 2 .
All the Christoffel symbols associated with the constrained connection are zero in terms of the $\mathbb{G}$-orthogonal generators

$$
\begin{aligned}
& X_{1}=\rho \cos \theta \frac{\partial}{\partial x}+\rho \sin \theta \frac{\partial}{\partial y}+\frac{\partial}{\partial \phi}, \\
& X_{2}=\frac{\partial}{\partial \theta} .
\end{aligned}
$$

Two input vector fields are considered for this system

$$
Y_{1}=\frac{1}{J_{\text {spin }}} X_{2}, \quad Y_{2}=\frac{1}{m \rho^{2}+J_{\text {roll }}} X_{1} .
$$

Let us consider different cases of input vector fields:

1) $Y_{1}$ only: The constrained affine connection restricts to Sym $^{(\infty)} Y_{1}=Y_{1}$. The system is not accessible from $v_{q} \in \operatorname{Sym}^{(\infty)} Y_{1}$ because of Theorem 4.5

2) $Y_{2}$ only: The constrained affine connection restricts to Sym ${ }^{(\infty)} Y_{2}=Y_{2}$. The system is not accessible from $v_{q} \in \operatorname{Sym}^{(\infty)} Y_{2}$ because of Theorem 4.5

3) $Y_{1}$ and $Y_{2}$ : The constrained affine connection restricts to $\operatorname{Sym}^{(\infty)}\left(Y_{1}, Y_{2}\right)_{q}=T_{q} \bar{Q}$. Using similar reasoning as for the planar rigid body, we conclude that the system is accessible and configuration accessible from $v_{q} \in \operatorname{Sym}^{(\infty)} \mathscr{Y}$.

In order to decide about the configuration accessibility for single-input case, we will have to compute $\operatorname{Lie}^{(\infty)}(Z, \mathscr{Y})$. In these particular cases computations show that the horizontal subspace of $\operatorname{Lie}^{(\infty)}\left(Z, \mathscr{Y}^{V}\right)$ is never the entire tangent space $T_{q} Q$. Thus the system is not configuration accessible from $v_{q} \in \operatorname{Sym}^{(\infty)} \mathscr{Y}$ when $\mathscr{Y}=\left\{Y_{1}\right\}$ or $\mathscr{Y}=\left\{Y_{2}\right\}$.

\section{CONCLUSIONS AND FUTURE WORKS}

\section{A. Conclusions}

In this paper we have generalized results about the accessibility for mechanical control systems called affine connection control systems that were proved in [5]. Precisely, Theorem 2.3 has been extended to Theorem 4.5. The latter result characterizes intrinsically the accessibility properties of a mechanical control system at points with velocities in $\operatorname{Sym}^{(\infty)} \mathscr{Y}$ if $\nabla$ restricts to $\operatorname{Sym}^{(\infty)} \mathscr{Y}$.

First we have identified the primitive brackets at nonzero velocity in Theorem 3.1. This result and Proposition 4.1 have allowed us to characterize the accessibility distribution at points in $\operatorname{Sym}^{(\infty)} \mathscr{Y}$ as long as $\nabla$ restricts to $\operatorname{Sym}^{(\infty)} \mathscr{Y}$, see Corollary 3.2. Propositions 4.3 and 4.4

\section{B. Future Works}

It still remains to extend Theorem 4.5 to any velocity point under the assumption of having $\nabla$ restricted to $\operatorname{Sym}^{(\infty)} \mathscr{Y}$ and also discarding this assumption. Once we succeed to completely characterize the accessibility algebra, our efforts will focus on extending the characterization of controllability in the sense of STLC given in [5] at points with non-zero velocity, cf. Definition 2.1

It is expected that infinitesimal holonomy algebra, cf. [3], will play an important role for these extensions. 


\section{ACKNOWLEDGMENTS}

The author gratefully acknowledges Professor A. D. Lewis for useful discussions.

\section{REFERENCES}

[1] F. Bullo, A. D. Lewis, Geometric Control of Mechanical Systems. Modeling, analysis and design for simple mechanical control, Texts in Applied Mathematics 49, Springer-Verlag, New York-HeidelbergBerlin 2004.

[2] F. Bullo, A. D. Lewis, Supplementary Chapters of Geometric Control of Mechanical Systems. Modeling, analysis and design for simple mechanical control, Texts in Applied Mathematics 49, SpringerVerlag, New York-Heidelberg-Berlin 2004.

[3] S. Kobayashi, K. Nomizu, Foundations of Differential , volume 1 of Tracts in pure and applied mathematics. Interscience Publishers, New York, London, 1964.

[4] A. D. LEWIS, Affine connections and distributions with applications to nonholonomic mechanics. Pacific Institute of Mathematical Sciences Workshop on Nonholonomic Constraints in Dynamics (Calgary, AB, 1997). Rep. Math. Phys. 42 (1998), no. 1-2, 135-164.

[5] A. D. LEwis, R. M. MurRay, Configuration Controllability of Simple Mechanical Control Systems, SIAM Journal on Control and Optimization, 35(3)(1997), 766-790.

[6] E. Martínez, J. F. Cariñena, W. Sarlet, Derivations of differential forms along the tangent bundle projection, Differential Geom. Appl. 2(1)(1992), 17-43.

[7] E. Martínez, J. F. Cariñena, W. Sarlet, Derivations of differential forms along the tangent bundle projection. II, Differential Geom. Appl. 3(1)(1993), 1-29.

[8] H. NiJMEIJER, A. J. VAN DER SCHAFt. Nonlinear Dynamical Control Systems, Springer-Verlag, New York, Heidelberg, Berlin, 1990.

[9] H. J. SussmanN, V. JuRdjevic. Controllability of nonlinear systems, J. Differential Equations, 12(1972), pp. 95-116.

[10] K. YANO AND S. IshiHARA. Tangent and cotangent bundles: differential geometry. Marcel Dekker Inc., New York, 1973. Pure and Applied Mathematics, No. 16. 